\title{
Smelling via the mouth: Effect of aging
}

\author{
JOSEPH C. STEVENS and WILLIAM S. CAIN \\ John B. Pierce Foundation Laboratory and Yale University, New Haven, Connecticut
}

\begin{abstract}
A group of 20 elderly subjects (67-83 years old) and a group of 20 young subjects (18-24 years old) made magnitude estimations of $\mathrm{NaCl}$ and ethyl butyrate solutions, orally sampled, first with the nose unpinched, then with the nose pinched shut to prevent the airborne (volatile) molecules from reaching the olfactory receptors via the nasopharynx. In the concentrations studied, ethyl butyrate had weak taste, strong odor. On the basis of earlier work, which has shown widespread age-related hyposmia, it was hypothesized that pinching the nose would lower the perceived intensity of ethyl butyrate more in young than in old subjects. In order to discount individual inclinations to use large or small numbers, the $\mathrm{NaCl}$ estimations were used to normalize the ethyl butyrate estimations. It then became clear that the average young subject perceived the overall intensity of ethyl butyrate solutions to be much stronger with the nose unpinched; for the elderly, in contrast, it made little difference whether the nose was pinched or unpinched. Olfactory losses inferred in this study are of comparable magnitude to those measured in earlier studies, in which the odorants were sniffed via the nostrils.
\end{abstract}

The perception of food and beverages involves several sense modalities: taste, touch, temperature, proprioception, olfaction, common chemical sense, even hearing (Vickers, 1985). The olfactory component comes about largely by airborne molecules that make their way to the olfactory receptors via the nasopharynx. A striking feature of this component is its frequent "referral" to the mouth; that is, odors aroused through the nasopharyngeal route are frequently attributed to taste (Murphy \& Cain, 1980; Murphy, Cain, \& Bartoshuk, 1977). Pinching the nostrils shut blocks the olfactory stimulation and thereby eliminates the referral. The purpose of the present study was to compare nasopharyngeal smelling in young and old persons. Three considerations motivated this endeavor.

First, there is no question that aging frequently weakens the sense of smell. This shows up in higher detection thresholds (for reviews of numerous studies see Murphy, 1986; Schiffman, 1979), diminished suprathreshold intensity (Stevens, Bartoshuk, \& Cain, 1984; Stevens \& Cain, 1985, 1986; Stevens, Plantinga, \& Cain, 1982), impaired ability to identify and discriminate odors (Doty et al., 1984; Schemper, Voss, \& Cain, 1981; Schiffman \& Leffingwell, 1981), and impaired ability to identify foods (Murphy, 1985; Schiffman, 1977).

Second, the cause or causes of age-related hyposmia are still undetermined. One possibility is that the airways in the vicinity of the olfactory mucosa become partially blocked; such blockage could conceivably blunt olfactory response via the nostrils more than that via the mouth. One reason for suspecting this possibility was the infrequency with which our many elderly, noninstitutionalized,

This research was supported by NIH Grant No. AG04287. The authors thank Amy Plantinga Pauw for extensive technical assistance. Address correspondence to Joseph C. Stevens, John B. Pierce Foundation Laboratory, 290 Congress Ave., New Haven, CT 06519. hyposmic subjects complained about the quality of their food perception. This subject is discussed further below.

Third, Schiffman (1977) reported that young subjects could identify common foods, pureed to eliminate texture cues, much better than could old subjects. Murphy (1985) confirmed this finding; however, when the stimuli were sampled with the nose pinched shut the performances of the young and old were approximately the same. Murphy concluded that the impairment in old age is more readily ascribable to olfactory (and possibly trigeminal) weakening than to gustatory weakening. This conclusion is bolstered by our own finding, several times replicated, that age takes a greater toll on olfactory and trigeminal magnitude than on gustatory magnitude; however, in our experiments the stimuli were delivered by way of the nostrils (Bartoshuk, Rifkin, Marks, \& Bars, 1986; Stevens et al., 1984; Stevens \& Cain, 1985, 1986; Stevens et al., 1982).

In the present study we compared the suprathreshold magnitude of ethyl butyrate sampled orally with the nose pinched and with the nose unpinched. This compound was chosen because it is a potent olfactory stimulus (resembling fruit-flavored chewing gum), but has little taste.

\section{METHOD}

\section{Subjects}

The subjects were 20 persons aged $67-83$ (mean $=74.4, \sigma=4.7$ ) and 20 persons aged $18-24$ (mean $=20.9, \sigma=1.59$ ). The groups were balanced for sex (although no sex difference emerged). The elderly subjects were tested at two senior-citizen centers, the young in the laboratory. All were required to be nonsmokers and to be able to breathe freely through the nostrils.

\section{Procedure and Stimuli}

In the course of a test session, a subject made magnitude estimations of five concentrations of liquid solutions of ethyl butyrate and $\mathrm{NaCl}$ on a common scale of sensory strength. This procedure, called magnitude matching (Stevens \& Marks, 1980), has been used several 
times in the above-mentioned studies comparing olfactory, trigeminal, and gustatory suprathreshold intensity. In the first half of the test session, the $\mathrm{NaCl}$ and ethyl butyrate concentrations were estimated with the nose unpinched. After a 5- to 10-min rest, the subject estimated the same stimuli with his/her nose pinched by a swimmer's clip (or, if the clip caused significant discomfort, with the hand). The stimuli (about $10 \mathrm{ml}$ ) were sipped from small plastic cups and expectorated, with rinses in between with deionized water. The solutions were made up of reagent-grade chemicals and dionized water. Their concentrations were as follows: for $\mathrm{NaCl}, 0.02,0.035$, $0.063,0.112$, and 0.20 molar; for ethyl butyrate, $0.0037 \%$, $0.0065 \%, 0.012 \%, 0.021 \%$, and $0.037 \%$ by volume. There were two runs of these levels in both halves of the session (four runs total). Ethyl butyrate and $\mathrm{NaCl}$ were alternated from trial to trial; the levels came in random order for any run, except that, as a partial control on adaptation, the lowest level never immediately followed the highest level. Each run also contained two blank stimuli (water only). Stimuli were given at the rate of one every 30-45 sec.

Before the magnitude matching began, the subject was asked to make magnitude estimations of the span between the experimenter's forefingers, from zero to maximal arm stretch. This acquainted the subject with magnitude estimation and ensured that the subject understood that large, small, zero, and decimal numbers were permissible. Then the experimenter gave the following instructions, in paraphrased form, while maintaining eye contact with the subject:

Now you are going to make a similar kind of judgment about the strength of certain tastes. You will taste from these little cups [demonstrated]. The stronger the solution tastes or smells, the bigger the number you should give to it. Zero would mean that you tasted or smelled nothing. You may find that you need to use zero frequently. If so, do not worry about it. Just judge the way it seems to you. Remember that you can use decimals, fractions, low and high numbers, just as you could in judging lengths. Try to judge all the solutions on the same scale. Thus, if one fruity taste should happen to seem equal to a salty taste, you would give them the same number. However, it may seem to you that the salty solutions are usually greater than the fruity solutions, or the other way round. So it is important to avoid assuming that the same sizes and ranges of numbers should be used for both.

After this instruction, a few practice trials were given (no feedback), including blanks. Blanks were identified as zero and the subject was told that these would sometimes be presented in the course of testing.

\section{RESULTS}

Several studies using magnitude matching have yielded high correlations between the sizes of the numbers used by the subjects to estimate the two (or more) continua. That is, a subject who tends to estimate salts with large (or small) numbers tends also to estimate ethyl butyrate with large (or small) numbers. The salt continuum furnishes a control on the individual inclination to use a particular number range. The estimates of ethyl butyrate were, accordingly, normalized by means of the salt estimates: every estimate of a given subject was multiplied by a factor that made the mean taste estimate equal 10 . The mean normalized salt estimates are plotted in Figure 1, which shows that the form of the functions for young and old differed little.

The mean normalized estimates of ethyl butyrate are plotted in Figure 2, which shows that the young estimated

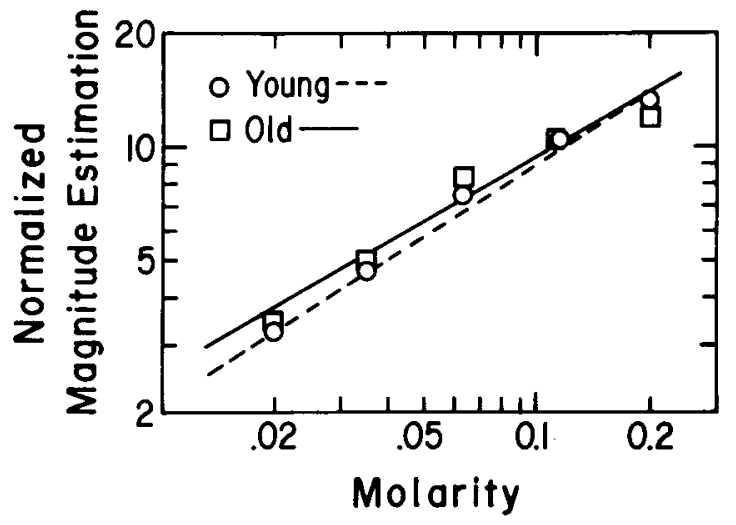

Figure 1. Comparison of mean magnitude estimations of the strength of $\mathrm{NaCl}$ by young and old subjects.

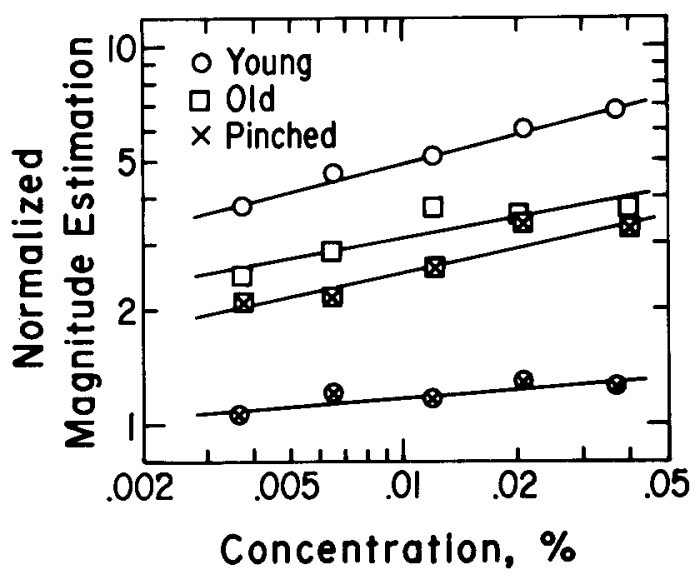

Figure 2. Comparison of mean magnitude estimations of the strength of ethyl butyrate by young and old subjects. The solutions were sampled with and without the nose pinched shut.

these stimuli to be much stronger with the nose unpinched than with the nose pinched. At each concentration level, the difference between the means is significant (.00005 $<p<.0002$ by paired $t$ tests). In contrast, the difference between pinched and unpinched conditions for the elderly was significant only at the middle $(0.012 \%)$ concentration $(p<.05)$. The functions of the elderly are also more erratic than those of the young. That the elderly subjects' function for the pinched-nose condition has a steeper slope and higher position than that of the young subjects probably reflects response biases that are virtually inevitable in an experiment that applies the same stimulus conditions to two groups whose sensitivities differ radically. Elevated slopes result when the subjective range of the stimuli is very small (Teghtsoonian, 1973). Also, in experiments on olfaction, the elderly tend to emit many false positive responses to stimulus blanks (Stevens \& Cain, 1985, 1986) and to stimuli below the individual detection threshold (Stevens \& Cain, in press), often substantial relative to their other estimates. In the present experiment, too, the elderly gave more and larger 
false positives than the young. The net effect of high falsepositive responding may have been to elevate the position of the psychophysical function. Or it may simply be that the young subjects were so struck by the contrast between the sensations with nose pinched and nose unpinched that they felt very small numbers were called for in the second half of the testing. Most young subjects spontaneously expressed great surprise at the magnitude of the "taste" change brought about by pinching the nose (the reader can verify the feeling by painting a few drops of vanilla on the tongue with nose pinched, then releasing the nose). In contrast, only one elderly subject mentioned the change.

Another way to inspect the data is to compare for young and old the differences between the normalized estimates of the same ethyl butyrate levels made with the nose pinched and with the nose unpinched. These differences appear in Figure 3. They are large and systemataic for the young, small and erratic for the old. At each concentration level, the two points differ significantly (.00008 $<p<.003$ by $t$ tests). Differences based on raw (nonnormalized) data also all differ significantly $(.00004<p<.008)$.

Figure 4 conveys information about individual performances. Each point is the ratio of a subject's mean estimate of ethyl butyrate to the subject's mean estimate of $\mathrm{NaCl}$ (a statistic we have called the index $i$ of olfactoryto-taste sensitivity in earlier studies). As in our earlier studies, cited above, this index varies widely across subjects; just how much of this variation represents real sensory variation and how much response criterion and instability (both numerator and denominator are subject to error of the random type) is impossible to say. For the present purpose, however, the mean $i$ (arrows) does reflect the main features of the outcome, and, as will become clear, permits a valuable quantitative comparison with olfactory studies based on sniffing through the nostrils (see

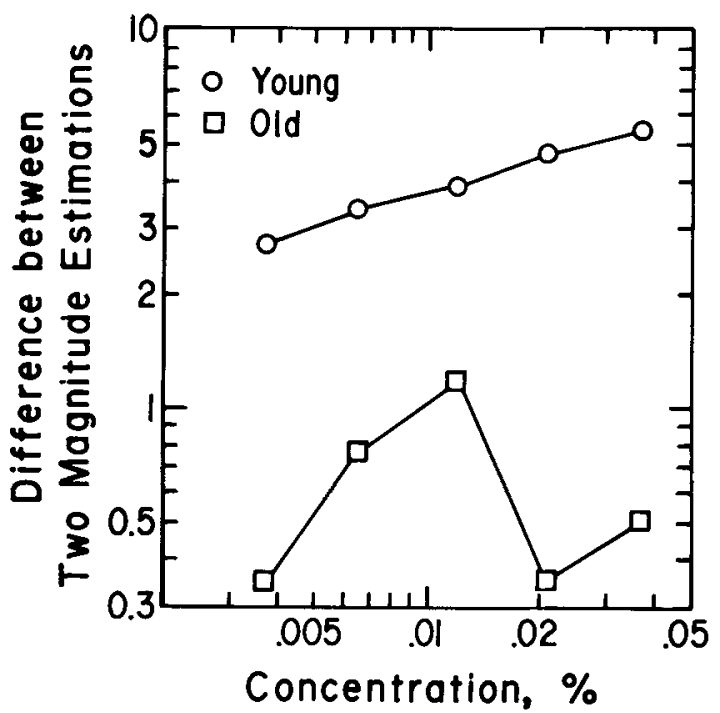

Figure 3. Comparison, for young and old subjects, of the mean difference of the magnitude estimations with and without the nose pinched shut.

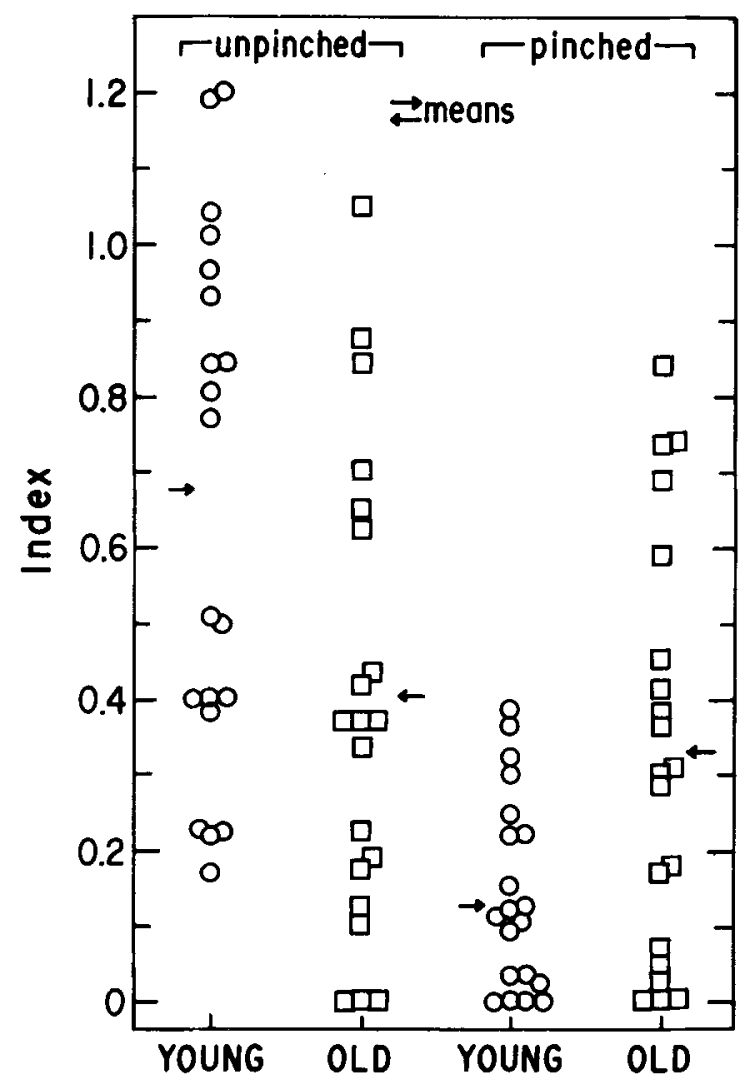

Figure 4. The index $i$ of relative estimated strength of ethyl butyrate to $\mathrm{NaCl}$, for each subject and for pinched and unpinched conditions.

Discussion). For the nose-unpinched condition, the mean is significantly larger for the young ( $p<.006$ by $t$ test) For the nose-pinched condition, the ratio is significantly higher for the old $(p<.005)$, again probably reflecting the response bias discussed above. On the other hand, for the young the mean ratio for the pinched condition is much smaller than for the unpinched condition $(p<.000006)$. In contrast, for the old the mean ratios for the pinched and unpinched conditions are practically identical.

It is also of interest and significance that the Pearson correlation coefficients relating the indices for the pinched and unpinched conditions were nonsignificant $(r=-.01)$ for the young subjects and significant for the old subjects $(r=.61)$. In all probability, this reflects the fact that the elderly subjects' indices were in both conditions governed primarily by oral sensations. Removal of the relatively weak olfactory cues therefore had a relatively small influence on the index. In contrast, the indices for the young rested heavily on the olfactory component, and removal of olfactory cues had drastic effects on the indices, rendering the correlation between the two conditions insignificant.

\section{DISCUSSION}

It is clear that aging takes a toll on nasopharyngeal smelling, just as it does on smelling by sniffing through 
the nostrils. The losses would appear to be of roughly equal magnitude, insofar as one can gauge by comparing the average indices $i$ from various studies. These studies used different groups (but approximately equal numbers) of subjects, with somewhat different average ages, and used different compounds and different measures of central tendency (means, medians, geometric means). Table 1 shows the average $i$ for the young divided by the average $i$ for the old for nine odorous compounds in seven studies. (The absolute sizes of $i$ are of little interest, since they depend heavily on exact choice of stimulus levels for both continua.) One can see that the average ratio from the present study falls close to the mean of the other 15 ratios.

In this and in earlier studies we have queried our subjects about their senses of taste and smell and whether they enjoy their food. It is well documented (Cohen \& Gitman, 1959) that elderly persons living in nursing homes complain frequently about food. It is unknown how much of this relates to the quality of the food itself, the social setting in which it is consumed, and the state of health and appetite, as opposed to a weakening of the senses, especially the chemical senses. In our experiments, we have found complaints among elderly persons who still live independently to be much less common. Almost all of our subjects have reported that they still enjoy food. The majority-even those who show marked hyposmiastate that their senses of taste and smell are good. A few are aware that their sense of smell has weakened. Those persons who complain that their food does not taste as good as it used to rarely explain the change in terms of the chemical senses. Rather, they offer explanations such as denture problems, the financial inability to buy the quality of foods they would like, concern over chemical additives in foods, and digestive problems.

In contrast, both young and elderly persons who lose the sense of smell relatively abruptly, for instance, after a nasal infection or a severe blow to the head, recognize their loss and express unhappiness about it (Ferris et al.,

Table 1

Average Index $\boldsymbol{i}$ for Young Subjects Divided by Average Index $i$ for Old Subjects Obtained for Nine Odorous Compounds in Seven Studies

\begin{tabular}{llllll}
\hline \multicolumn{1}{c}{ Compound } & & & & & \\
\hline iso-Amyl butyrate & $1.60^{\mathrm{a}}$ & $1.34^{\mathrm{b}}$ & $1.69^{\mathrm{b}}$ & $1.56^{\mathrm{c}}$ & $1.41^{\mathrm{d}}$ \\
Benzaldehyde & $1.78^{\mathrm{c}}$ & $1.36^{\mathrm{d}}$ & & & \\
d-Limonene & $1.07^{\mathrm{c}}$ & $1.81^{\mathrm{d}}$ & & & \\
Pyridine & $1.66^{\mathrm{c}}$ & & & & \\
Ethyl alcohol & $1.63^{\mathrm{c}}$ & & & & \\
iso-Amyl alcohol & $1.37^{\mathrm{c}}$ & & & & \\
Ethyl mercaptan & $1.56^{\mathrm{c}}$ & & & & \\
Butanol & $1.52^{\mathrm{f}}$ & & & & \\
Ethyl butyrate & $1.47^{\mathrm{g}}$ & & & & \\
Mean & 1.52 & & & & \\
Standard deviation & 0.186 & & & & \\
\hline
\end{tabular}

aStevens et al., 1982; 'Stevens et al., 1984; ' Stevens \& Cain, 1985; ${ }^{\mathrm{d} S}$ Stevens \& Cain, in press; 'Stevens et al., 1986; ' Stevens \& Cain, 1986; spresent study, nose-unpinched condition.
1985). Thus, abrupt, rather than gradual, loss would seem to be the key factor that determines dissatisfaction with food.

Does this mean that the gradual weakening of the sense of smell has no bearing on the quality of life in old age? Not necessarily. For one thing, with weakened smell a person is less likely to detect gas leaks (Chalke \& Dewhurst, 1957; Stevens, Cain, \& Weinstein, 1986), smoke, and spoiled foods. For another, the observation by Schiffman (1977) that food perception deteriorates in old age led her to examine the effects of fortifying foods to various extents with artificial flavors. On the average, the older subjects in that study rated as better foods that were fortified to the right degree. As one might expect, the young subjects preferred unfortified foods. Thus we see that although gradual sensory losses can go unnoticed, abrupt improvements can be appreciated. It seems, for both nutritional and sensory reasons, that greater appeal can be made to the olfactory system and to the trigeminal sense, which is also subject to serious weakening in old age (Stevens \& Cain, 1986; Stevens et al., 1982). Appeals to the gustatory sense are likely to be less effective, and, in the case of salt and sweet, could be nutritionally unwise.

\section{REFERENCES}

Bartoshuk, L. M., Rifkin, B., Marks, L. E., \& Bars, P. (1986). Taste and aging. Joumal of Gerontology, 41, 51-57.

Chalke, H. D., \& Dewhurst, J. R. (1957). Accidental coal-gas poisoning: Loss of sense of smell as a possible contributory factor with old people. British Medical Journal, 2, 915-917.

COHEN, T., \& GitmaN, L. (1959). Oral complaints and taste perception in the aged. Joumal of Gerontology, 14, 294-298.

Doty, R. L., Shaman, P., Applebaum, S. L., Giberson, R., SiKSORSKI, L., \& ROSENBERG, L. (1984). Smell identification ability: Changes with age. Science, 226, 1441-1443.

Ferris, A. M., Schlitzer, J. L., Schierberl, M. J., Catalanotto, F. A., Gent, J., Peterson, M. G., Bartoshuk, L. M., Cain, W. S., Goodspeed, R. B., Leonard, G., \& Donaldson, J. O. (1985). Anosmia and nutritional status. Nutrition Research, 5, 149-156.

MURPHY, C. (1985). Cognitive and chemosensory influences on agerelated changes in the ability to identify blended foods. Journal of Gerontology, 40, 47-52.

MurPhy, C. (1986). Taste and smell in the elderly. In H. L. Meiselman \& R. S. Rivlin (Eds.), Clinical measurement of taste and smell. New York: Macmillan.

MURPHY, C., CAIN, W. S. (1980). Taste and olfaction: Independence vs. interaction. Physiology \& Behavior, 24, 601-605.

MurPhy, C., Cain, W. S., \& BartoshuK, L. M. (1977). Mutual action of taste and olfaction. Sensory Processes, 1, 204-211.

Schemper, T., Voss, S., \& CAIN, W. S. (1981). Odor identification in young and elderly persons: Sensory and cognitive limitations. Journal of Gerontology, 36, 446-452.

Schiffman, S. (1977). Food recognition by the elderly. Journal of Gerontology, 32, 586-592.

SCHIFFman, S. (1979). Changes in taste and smell with age: Psychophysical aspects. In J. M. Ordy \& K. Brizzee (Eds.), Sensory systems and communication in the elderly (Aging, Vol. 10, pp. 227-246). New York: Raven Press.

Schiffman, S., \& Leffingwell, J. C. (1981). Perception of odors of simple pyrazines by young and elderly subjects: A multidimensional analysis. Pharmacology, Biochemistry, \& Behavior, 14, 787-798. 
Stevens, J. C., Bartoshux, L. M., \& Cain, W. S. (1984). Chemical senses and aging: Taste versus smell. Chemical Senses, 9, 167-179.

Stevens, J. C., \& Cain, W. S. (1985). Age-related deficiency in the perceived strength of six odorants. Chemical Senses, 10, 517-529.

Stevens, J. C., \& CaIN, W. S. (1986). Aging and the perception of nasal irritation. Physiology \& Behavior, 37, 323-328.

Stevens, J. C., \& CaIN, W. S. (in press). Old-age deficits in the sense of smell as gauged by thresholds, magnitude matching, and odor identification. Psychology \& Aging.

Stevens, J. C., Cain, W. S., \& Weinstein, D. E. (1986). Aging impairs the ability to detect gas odor. Manuscript submitted for publication.

SteVens, J. C., \& Marks, L. E. (1980). Cross-modality matching func- tions generated by magnitude estimation. Perception \& Psychophysics, 27, 379-389.

Stevens, J C., Plantinga, A., \& Cain, W. S. (1982). Reduction of odor and nasal pungency associated with aging. Neurobiology of Aging, 3, 125-132.

TEGHTSOONIAN, R. (1973). Range effects in psychophysical scaling and a revision of Stevens' law. American Journal of Psychology, 86, 3-37.

VICKERS, Z. M. (1985). The relationships of pitch, loudness, and eating technique to judgments of the crispness and crunchiness of food sounds. Journal of Texture Studies, 16, 85-95.

(Manuscript received February 20, 1986; revision accepted for publication June 17, 1986.) 